\title{
IMPROVING DEPARTURE THROUGHPUT BY DYNAMICALLY ADJUSTING INTER-ARRIVAL SPACING
}

\author{
Hyo-sang Yoo ${ }^{1}$, Paul Lee $e^{3}$, San Jose State University Research Foundation/NASA Ames Research \\ Center, Moffett Field, CA \\ Everett Palmer ${ }^{2}$, NASA Ames Research Center, Moffett Field, CA
}

\begin{abstract}
LaGuardia airport (LGA) in New York has many unique challenges that create excess taxi-out delays. The purpose of this paper is to investigate the potential benefit that could be gained by tactically adjusting the Terminal Sequencing and Spacing (TSS) schedule to precisely manage inter-arrival spacing to maximize the number of departures per arrival pair. Three strategies for dynamically adjusting arrival schedules are proposed in this paper: Delay Control, Delay and Advance, and No Slack Capacity. The benefits of these strategies were examined on actual traffic data at LGA. The results showed that by applying these strategies, a 10 to $60 \%$ increase in departures and a reduction in unutilized departure capacity (gaps) could be achieved during the airport's busiest six-hour period. Significant increases in departure throughput would improve air traffic operations by reducing departure delay time. Furthermore, the concept could be used to resolve temporal mismatches between departure capacity and demand which also cause excessive departure delays.
\end{abstract}

\section{Introduction}

In today's air traffic operations, taxi-out delays account for the largest proportion of aviation movement delay [1]. Taxi-out delays can negatively impact the direct operational costs to the airlines by increasing fuel cost and schedule uncertainty. The associated schedule uncertainty can also have further reaching effects beyond the airport

Taxi-out delays can be caused by a variety of factors such as weather and taxiway congestion. For example, a weather cell can block a departure gate, temporarily impeding departures and creating a long departure queue. The weather cells can also delay the arrivals such that a pent-up arrival demand exists. These aircraft then need to land once the weather clears. The prioritization of arrivals under these conditions creates a situation in which the departure operations are temporarily suspended, which in turn creates departure delays. Regardless of the causal factors, the resulting outcome is an increase in the taxi-out delays due to an imbalance between departure demand and departure capacity.

Recently, research has been conducted to explore ways to improve departure operations and reduce delays without significantly impacting arrival throughput. One promising approach has been to implement an integrated departure-arrival schedule. For example, Diffenderfer and Osburn prototyped and evaluated a tool that can tactically adjust and display inter-arrival spacing with gaps for multiple departures [2 and 3]. Although the concept and the tools were promising, the results suggested that lastminute, tactical adjustments of the arrival schedule were less effective than initially hypothesized.

Therefore, the present study has extended this concept to allow the TRACON controllers to identify and adjust the arrival schedule for departures when the arrivals enter the TRACON airspace. The scheduling is accomplished using features of the Terminal Sequencing and Spacing (TSS) system [4]. TSS is a collection of advanced time-based arrival scheduling management technologies combined with controller precision spacing tools [4].

Three tactical strategies for dynamically adjusting the schedules of arrival aircraft are identified. The strategies are designed to allow for the precise management of inter-arrival spacing to enable the departure of multiple aircraft per arrival while introducing minimal changes to arrival throughput. Next, these strategies are applied to the actual traffic data at LaGuardia (LGA) airport to determine how much benefit, in terms of increase in departure throughput, could be achieved 
The rest of the paper is organized to describe 1) the departure delay problems at LGA airport, 2) the concept for dynamically adjusting arrival schedules, and 3) the assessment of the concept on actual traffic data to estimate the potential benefit.

\section{Background}

\section{Departure Delay Problems at LGA airport}

New York (NY) airspace has been identified as the most congested in the United States. It serves three major airports-John F. Kennedy International Airport (JFK), Newark Liberty International Airport (EWR) and LGA. These airports have very high traffic demand within close proximity of each other, creating airspace challenges unparalleled in the United States. The NY region accounts for a large portion of all US traffic delays, and these delays in turn ripple across the U.S. [5].

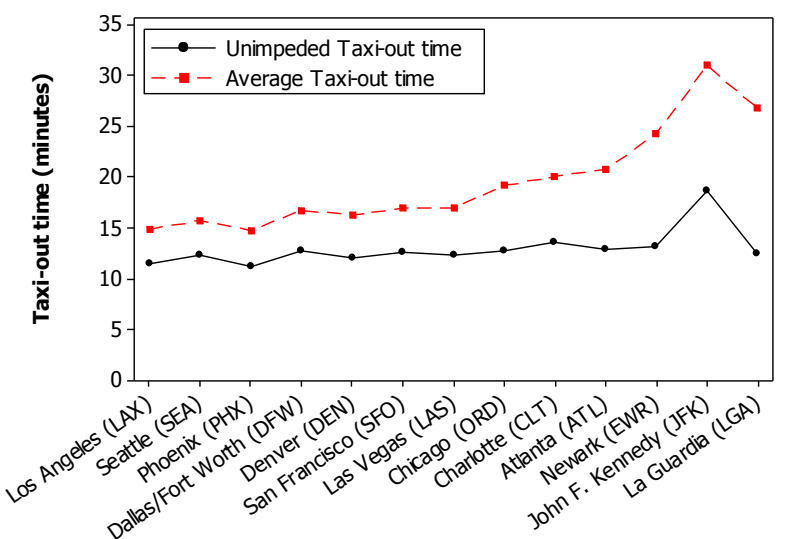

Figure 1. Taxi-Out Time between 2007 and 2012.

When the types of delays experienced in the National Airspace System (NAS) are categorized by the different phases of flights (e.g., airborne, gate, taxi-out), taxi-out delays account for a large portion of the overall delays. The plots in Figure 1 are generated from data reported in the Aviation System Performance Metrics (ASPM) database which is maintained by the Federal Aviation Administration (FAA). The figure shows the Taxi-Out Time between 2007 and 2012 at the three primary airports in New York and the other 10 busiest US airports. Figure 1 shows that among the busiest airports in the United States, LGA has the largest discrepancy between the unimpeded taxi-out time and the average taxi-out time. Hence, for this study, LGA traffic was chosen for further analyses and concept evaluation.
A closer look at LGA operations showed a number of unique challenges that created excess taxiout delays. The LGA airport has intersecting runways such that the departure and arrival operations are interdependent. It also has a limited number of taxiways, which creates long and inflexible departure queues. Finally, from morning to evening each day, the airport runs at near maximum departure and arrival capacity. Hence, delays accumulate throughout the day with even small mismatches between departure demand and departure capacity. These mismatches can be due to small operational miscalculations such as a missed departure slot due to tight inter-arrival spacing.

Figure 2 shows a screen-capture from an Airport Surface Detection Equipment-Model X (ASDE-X). The figure shows a departure queue that has grown to the point of impeding an efficient departure flow. Increasing departure capacity over demand would lessen this problem, reducing the departure queues.

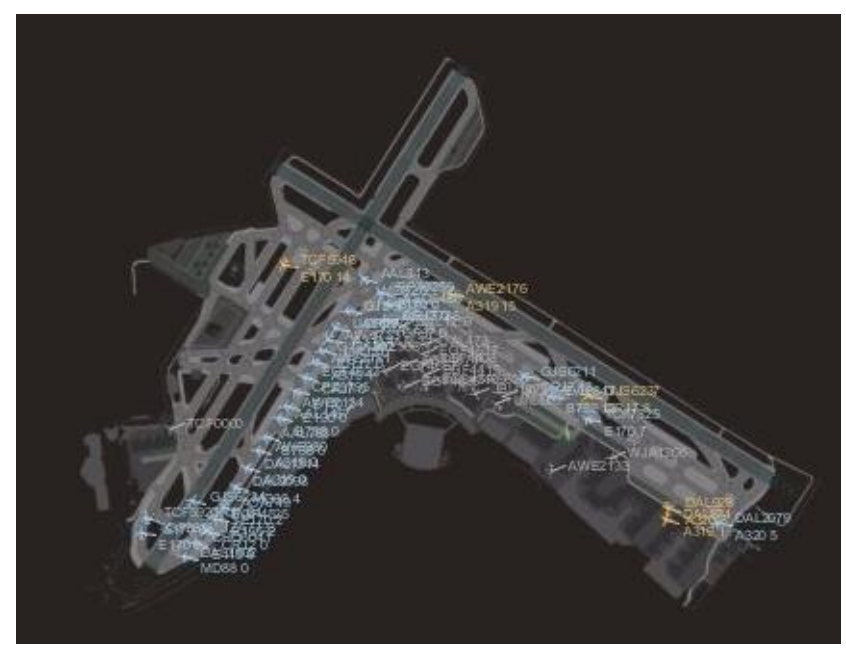

Figure 2. 31|4 RWY operations on 04/05/2013

\section{Unutilized (Slack) Departure Capacity in Current-day Operations}

As mentioned, one of the contributing factors to departure delay is excessive departure demand. In this paper, departure demand is defined as the number of aircraft scheduled to depart from an airport during a specified time interval. Departure capacity is defined as the maximum number of aircraft that an airport can depart during a specified time interval. 
The departure queue continues to grow as long as the mismatch between demand and capacity exists, which increases departure delays. One way to resolve this problem is by increasing departure capacity. At LGA, the intersecting runways limit the number of departures relative to the number of arrivals. During a busy traffic period, only one departure can be cleared for takeoff per arrival, with occasional opportunities to clear multiple departures between arrivals when the inter-arrival spacing is sufficiently large.

Figure 3 depicts the inter-arrival spacing of 25 arrival aircraft sampled from actual traffic flows at LGA airport on April 21, 2013. On that day, LGA was operating in a RWY 22|13 (landing runway 22 and departing runway 13) configuration under Visual Meteorological Conditions (VMC). The plots are generated based on Center-TRACON Automation System (CTAS) data maintained by NASA. In Figure 3 , each bar represents an arrival aircraft and the height of each bar indicates the temporal spacing to the trailing aircraft in seconds.
In current day operations, one departure per arrival pair (one for one) is the norm. If you assume that 70 seconds is the minimum required inter-arrival spacing for one aircraft to depart, the plots in Figure 3 show the presence of wasted or unused capacity (to be referred to as slack capacity in the rest of paper). For example, Figure 3 shows that the first aircraft has 115 seconds spacing to the trailing aircraft-this indicates that there is $45(115 \mathrm{~s}-70 \mathrm{~s})$ seconds of slack capacity.

As illustrated in Figure 3, the inter-arrival spacing is loosely managed, thereby creating less than optimal spacing for departures. In this paper, we describe approaches to access the unused departure capacity by better managing the arrival schedule. A goal is to reduce the excess inter-arrival spacing in some cases and to increase the spacing to allow multiple departures in other cases.

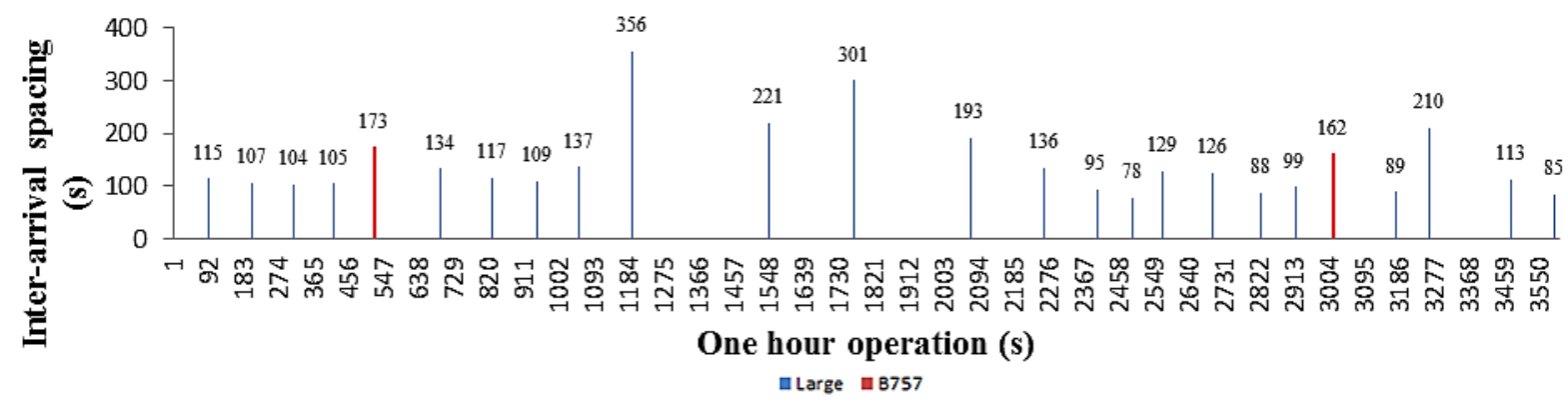

Figure 3. Inter-arrival Spacing in Seconds (s) - an Example of 25 Arrivals per Hour.

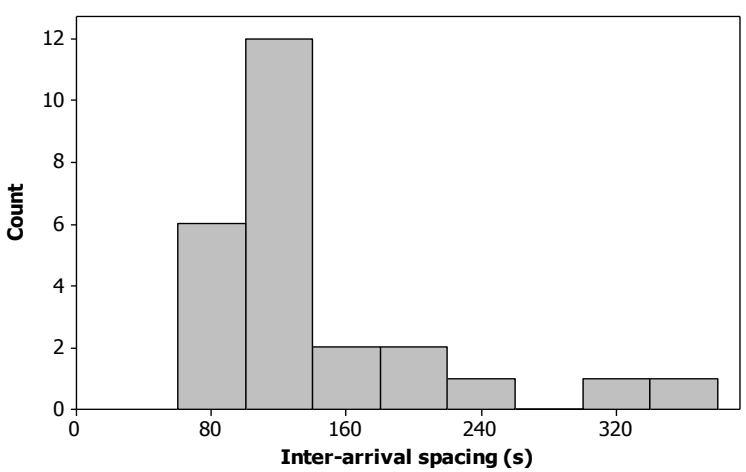

Figure 4. Histogram of Inter-Arrival Spacing in Seconds (s).
The presence of slack capacity in actual arrival traffic is apparent in Figure 4. Figure 4 shows the histogram of the 25 sampled arrivals' inter-arrival spacing that is depicted in Figure 3. The right tail in the histogram represents the cases where there is extra spacing in the arrival flow that could be used by departures.

Without any arrival schedule adjustments, multiple aircraft can depart opportunistically whenever there are pairs of arrivals with sufficiently large gaps between them. Assuming 60 seconds for each departure in a multiple-departure gap, there are twelve arrivals in Figure 3 with sufficient spacing for more than one departure. However, an active adjustment of the arrival schedule can yield an even greater number of departure slots. 


\section{Three Schedule Adjustment Strategies}

Three arrival schedule adjustment strategies have been identified that offer potential gains in the number of available departure slots: 1) Delay Control, 2) Delay and Advance Control, and 3) No Slack Capacity.

\section{Delay Control Strategy}

In this strategy, the arrival schedule is adjusted by delaying only the trailing aircraft and only if it does not cause a violation of minimum spacing to the following aircraft. The inter-arrival spacing of each pair of arrivals is evaluated to determine whether delaying the trailing aircraft could result in a sufficient gap for an additional aircraft. If so, the delay control is applied to the trailing aircraft.

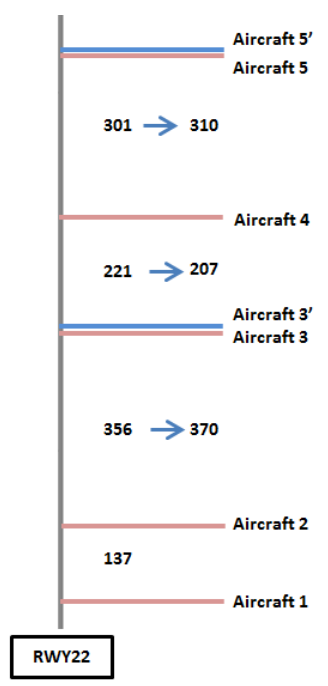

Figure 5. Example of Delay Control Strategy (Red $=$ Original Schedule, Blue $=$ Adjusted Schedule) .

Figure 5 illustrates how Delay Control could be applied. If we assume that the minimum required inter-arrival spacing for having one departure between two arrivals is 70 seconds, and 60 seconds is the time interval required to depart an additional departure, applying 14 seconds of Delay Control to Aircraft 3 could result in an extra departure, as the inter-arrival spacing changes from 360 to 370 seconds.

\section{Delay and Advance Control Strategy}

In this strategy, a time interval between arrivals is adjusted by advancing the leading aircraft and/or delaying the trailing aircraft. Advance Control can be applied to the leading aircraft only if speeding up the aircraft does not affect the slot that has been already created for an additional departure and also does not violate the minimum separation spacing to the aircraft that is flying ahead of it. Delay Control, the first strategy, is applied only when it does not create a separation violation to the trailing aircraft. We consider Advance Control to improve upon Delay Control in regards to fuel savings. Delay Control is considered only if Advance Control is not applicable.

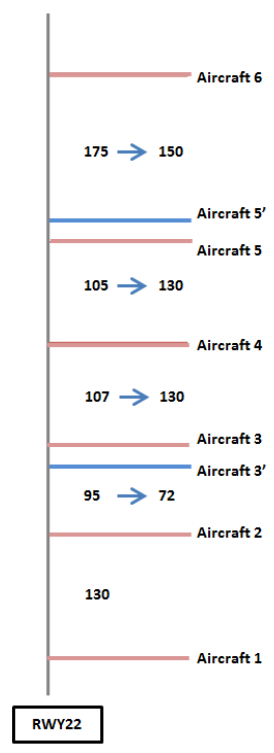

Figure 6. Example of Delay and Advance Control Strategy $($ Red $=$ Original Schedule, Blue $=$ Adjusted Schedule).

In Figure 6, Aircraft 3 is advanced (Advance Control) to create a sufficient gap for an additional departure. For the spacing between Aircraft 4 and Aircraft 5, Delay Control is applied, because advancing Aircraft 4 affects the departure slot (spacing between Aircraft 3 and Aircaft 4) that has been already created.

\section{No Slack Capacity Strategy}

The core idea of this strategy is to remove any slack capacity in the arrival flow to allow the greatest number of departures possible given a particular arrival demand. In this strategy, the trailing aircraft schedule is adjusted to provide necessary spacing for the maximum number of aircraft that could depart between them. For every pair of aircraft, the time intervals are evaluated to determine whether delaying 
the trailing aircraft can lead to a sufficient slot for an extra departure. If a slot cannot be achieved, then the trailing aircraft is advanced to remove any slack capacity.

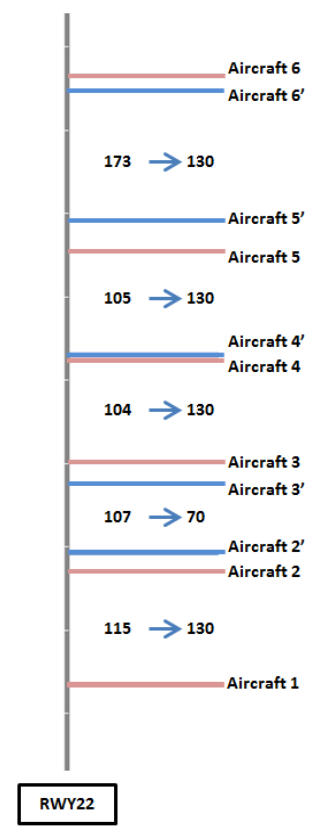

Figure 7. Example of No Slack Capacity Strategy $($ Red $=$ Original Schedule, Blue $=$ Adjusted Schedule).

In Figure 7, Aircraft 2 is delayed to create a sufficient gap for an additional departure, which affects the spacing between Aircraft 2 and Aircraft 3 . The time interval between Aircraft 2 and Aircraft 3 is not sufficient for an additional departure, even if Aircraft 4 is delayed. Hence, Aircraft 3 is advanced to remove any slack capacity.

In all three strategies, adjustment in schedule starts from the aircraft that is closer to landing first. For each strategy, 30 seconds was used as the limit on how much the controller could delay or advance the arrival schedule in order to create the necessary spacing for departures. We considered that control (speed and/or path control) applied within the feeder sectors of terminal airspace (i.e., the arrivals from the arrival fixes to the runway). Approximately 30 seconds delay or advance should be achievable by controllers with aircraft on Area Navigation (RNAV) routes. The 30 seconds limit is conservative; in actual operations, arrivals in the terminal airspace have fairly limited options in flying faster than their estimated times of arrival (ETAs) but can delay much longer than 30 seconds if necessary.

\section{Test Condition: LGA Airport}

In order to assess the benefit of the schedule adjustment strategies, actual traffic data from LGA was used. As a preliminary step to the analysis, the following parameters were applied to the input data: the runway configuration, the value of the required inter-arrival spacing for departures, and the time interval of a day.

\section{Runway Configuration}

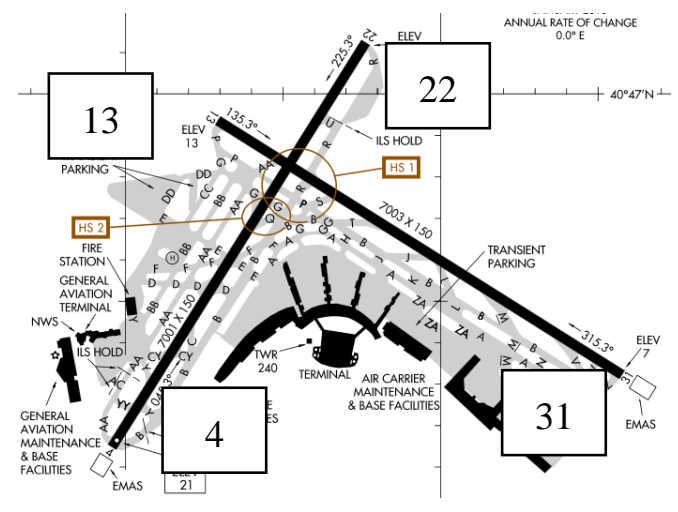

Figure 8. LaGuardia (LGA) Airport.

Based on ASPM data, it was determined that RWY 22|13 (landing runway 22 and departing runway 13 , see Figure 8 ) is the most frequently used configuration. Hence, we selected a day when RWY 22|13 was used and extracted the traffic data from the CTAS database.

\section{Required Inter-arrival Spacing for Departures}

The benefits available through the adjustment of arrival spacing are sensitive to the size of the required inter-arrival spacing for departures. The value of the parameters may change due to various factors, such as runway configuration, the type of the aircraft that lands/departs, weather conditions, etc. Nominally, the minimum required inter-arrival spacing for departures can be decomposed into three parts: Arrival-Departure (A - D) interaction, DepartureArrival $(\mathrm{D}-\mathrm{A})$ interaction, and Departure-Departure $(\mathrm{D}-\mathrm{D})$ interaction (see Figure 9). 


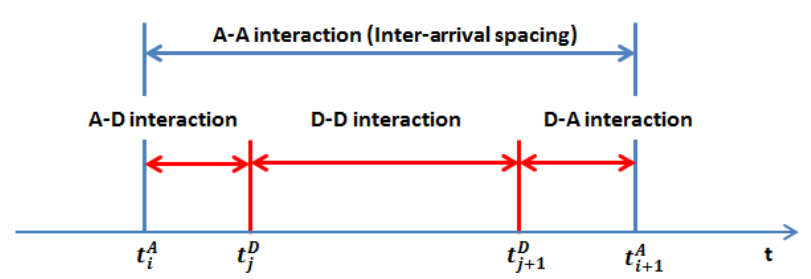

Figure 9. An Example of Two Departures Between Arrival Pair.

A - D interaction is the time interval between an arrival and a departure. In crossing operations, the arrival must clear the common intersection before the departure can be cleared for take-off. D - A interaction is the time interval between a departure and the next arrival. In crossing operations such as RWY 22|13, the departure must clear the common intersection prior to the arrival crossing the landing threshold. An additional factor that affects this time interval is wake turbulence restrictions, which may add additional spacing requirements. D - D interaction is the minimum required extra spacing for an additional departure, which is affected by the aircraft type and whether the successive departures are on divergent headings.

Based on nominal LGA operations, two assumptions were made to simplify the analysis. First, only large aircraft were included in the analysis because there are typically only a dozen B757 aircraft and even fewer Small aircraft per day at LGA. Second, no consecutive departure goes to the same departure fix, which is generally true during busy traffic hours.

Given these assumptions, the minimum required inter-arrival spacing $(d)$ for departures can be simply expressed as the following equation:

$$
d \cong a+b[n-1]
$$

In the equation above, $a$ indicates the minimum required inter-arrival spacing for one departure and it consists of a sum of $\mathrm{A}-\mathrm{D}$ and $\mathrm{D}-\mathrm{A} . \quad b$ represents the minimum required extra spacing for an additional departure, which is approximately equal to the wake vortex separation requirement. $n$ denotes the total number of aircraft that the controllers would like to depart between a pair of arrivals.

\section{Observational Study}

To identify the values of the illustrated parameters $a$ and $b$, an observational study of RWY $22 \mid 13$ operations was conducted using ASDE-X data from April 2, 2013. We recorded only the operations of large aircraft. Figure 10 shows the histogram of the parameter $a$ which is plotted based on the observed inter-arrival spacing values when there was only one departure between two arrivals. There were a total of 40 arrivals and 34 departures during an hour of VMC operations, with 28 inter-arrival spacing that we analyzed. These spacing had a mean of 87.8 seconds with a standard deviation of 17.2 seconds. The minimum value was 53 seconds and the maximum value was 120 seconds. The median is 89 seconds and the mode is 94 seconds.

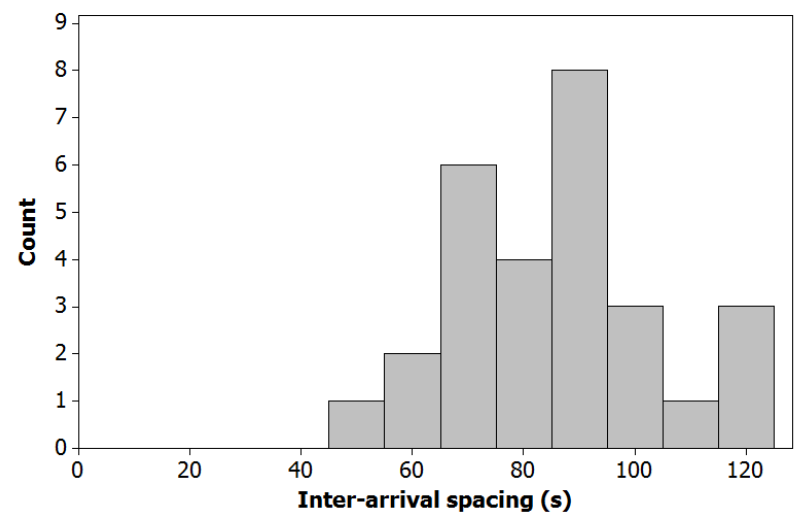

Figure 10. Histogram of Inter-Arrival Spacing (n $=28)$ in Seconds $(s)$.

The parameter $a$ is defined as the minimum required inter-arrival spacing for one departure. In the observational study, the value of the minimum inter-arrival spacing was observed to be 53 seconds. However, in the real world, this value may vary and is hard to standardize. We added one standard deviation ( $\mathrm{SD}=17$ seconds) to the minimum time we observed as possible idle time and chose 70 seconds as the value for the parameter $a$. This actually matches the second most frequently observed spacing in the observed data shown in Figure 10.

To determine the value of the parameter $b$, we searched the previous literature. One of the major factors that influences the time for a take-off clearance after a departure is wake vortex separation. When large aircraft type is followed by the same 
aircraft type, the first departure needs to be 6,000 feet down the runway and airborne prior to the take-off of the second departure [6]. Previous studies have investigated the required wake vortex separation between consecutive departures at different airports and identified the average wake vortex separations in seconds, as shown in Table 1 [7].

Table 1. Average Seconds Between Consecutive Departures Based on Wake Vortex Separation Requirements [7].

\begin{tabular}{|c|c|c|c|c|}
\hline & \multicolumn{4}{|c|}{ Leading Aircraft } \\
\hline $\begin{array}{c}\text { Trailing } \\
\text { Aircraft }\end{array}$ & Small & Large & Heavy & B757 \\
\hline Small & 59 & 88 & 109 & 110 \\
\hline Large & 59 & 61 & 109 & 91 \\
\hline Heavy & 59 & 61 & 90 & 91 \\
\hline B757 & 59 & 61 & 109 & 91 \\
\hline
\end{tabular}

The value of parameter $b$ is defined as the minimum wake vortex separation spacing in time between consecutive departures. Table 1 indicates that the average value is approximately 60 seconds for large aircraft. Hence, we set the required interarrival spacing $(d)$ for a number of departures between arrivals (n) to be $70+[60(n-1)]$ for the analysis in the latter part of the paper.

\section{Sensitivity to the Arrival Demand}

In order to increase the potential departure capacity by adjusting the arrival schedule, there must be sufficient gaps in the arrival demand to allow for schedule adjustments, i.e., if the arrivals are too tightly packed, then there will be no gaps for departure schedulers to use. Hence, we investigated whether the arrival rate at LGA airport provided sufficient natural slack to allow for additional departure capacity. Figure 11 is a box-plot of the arrival rate over the course of each day (00:00 23:00) at LGA for the 2013 fiscal year. This data only applies to when LGA was in the 22|13 configuration during $\mathrm{VMC}$, which shows that the time of the day had a significant impact on the arrival demand rate.

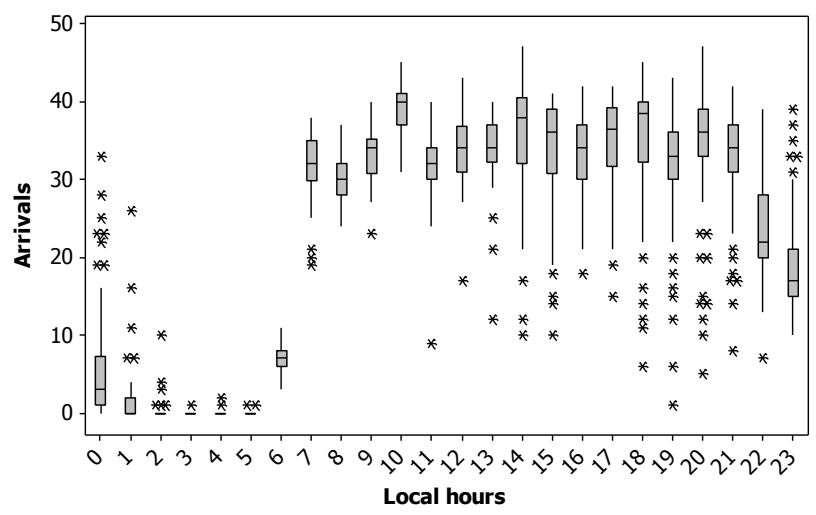

Figure 11. Box-Plot of Arrival Rate (00:00 23:00) for One Fiscal Year Operations (2013).

We categorized operations during a day into four time periods of 6 hours each and compared the arrival demand rate during each time period, as shown in Table 2. We found that on average, afternoon had the highest arrival rate.

Table 2. Summary Statistics of the Arrival Rate for One Fiscal Year of Operations (2013) at LGA

\begin{tabular}{|c|c|c|c|c|c|c|}
\hline & Mean & SD & Median & Max. & Min. & Mode \\
\hline $\begin{array}{c}\text { Morning } \\
(\mathbf{6 : 0 0}-\mathbf{1 1 : 5 9 )}\end{array}$ & 28.41 & 10.86 & 32 & 45 & 3 & 32 \\
\hline $\begin{array}{c}\text { Afternoon } \\
(\mathbf{1 2 : 0 0}-\mathbf{1 7 : 5 9 )}\end{array}$ & 33.97 & 6.32 & 35 & 47 & 10 & 38 \\
\hline $\begin{array}{c}\text { Night } \\
(\mathbf{1 8 : 0 0}-\mathbf{2 3 : 5 9})\end{array}$ & 29.53 & 9.36 & 32 & 47 & 1 & 39 \\
\hline $\begin{array}{c}\text { Late night } \\
(\mathbf{0 : 0 0}-\mathbf{5 : 5 9})\end{array}$ & 1.76 & 4.93 & 0 & 33 & 0 & 0 \\
\hline
\end{tabular}

From actual operations, we extracted the traffic data from the afternoon of 1/13/2014 (VMC) as a test busiest days. The rationale for choosing the busiest 
time period was because any departure throughput increase shown during that period would demonstrate the feasibility of the concept in the most challenging conditions and would indicate even greater improvements under lower arrival demand conditions.

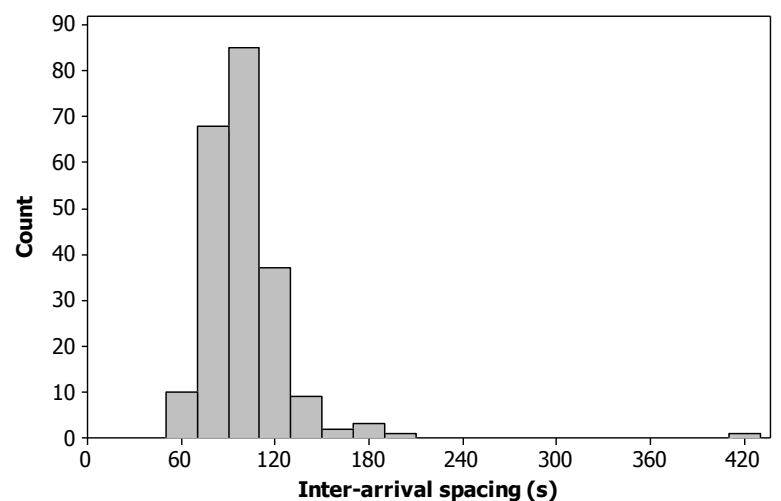

Figure 12. Histogram of Inter-Arrival Spacing in Seconds (s) during the Busiest 6-Hour Period.

Figure 12 presents a histogram of the interarrival spacing during the busiest 6-hour period we selected as the test condition. During these hours there were 216 arrivals and 209 departures. The interarrival spacing shown in Figure 12 has a sample mean of 100 seconds and a sample standard deviation of 30 seconds. The minimum value is 53 seconds and the maximum value is 413 seconds. The median is 95.5 seconds and the mode is 89 seconds.

\section{Benefit Assessment}

Using the inter-arrival spacing data from the busiest 6-hour period, the three strategies described in the earlier section (i.e., Delay Control, Delay and Advance Control, and No Slack Capacity) were applied to adjust the arrival schedule to increase the departure throughput. The results of the three strategies are compared to the One for One strategy, the current day method, and the Opportunistic approach, which is departing multiple aircraft opportunistically if there are sufficiently large gaps.

The results suggest that a significant increase in departures could be achieved with the three strategies described in this paper. Figure 13 shows the improvement in departure rate for each method for each one-hour period during the selected 6 hours. In comparison to the One for One and Opportunistic strategies, all three strategies show an improvement in departure rate. Such an increase in departure rate could lead to a reduction in departure delay.

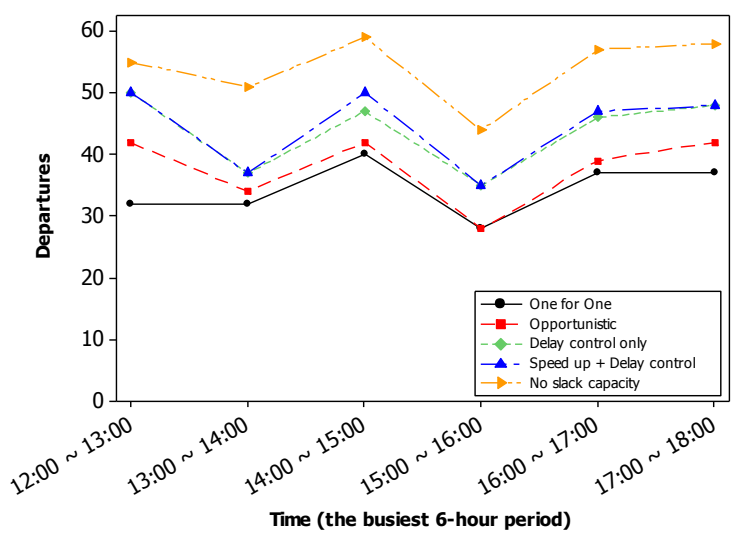

Figure 13. The Impact of the Different Strategies on the Number of Departures during the Busiest 6-Hour Period.

Figure 14 shows the increase in number of departures during the entire 6-hour period. There were a total of 216 arrivals during the selected period. During this period, the One for One strategy allows 206 aircraft to depart between arrivals. Using the Opportunistic strategy, 227 aircraft could depart. The resulting numbers of departures during the selected period for the three strategies were: 263,267 , and 324 respectively for Delay Control, Delay and Advance Control, and the No Slack Capacity strategies. These numbers are about a $10-60 \%$ increase compared to what could be achieved using the One for One approach.

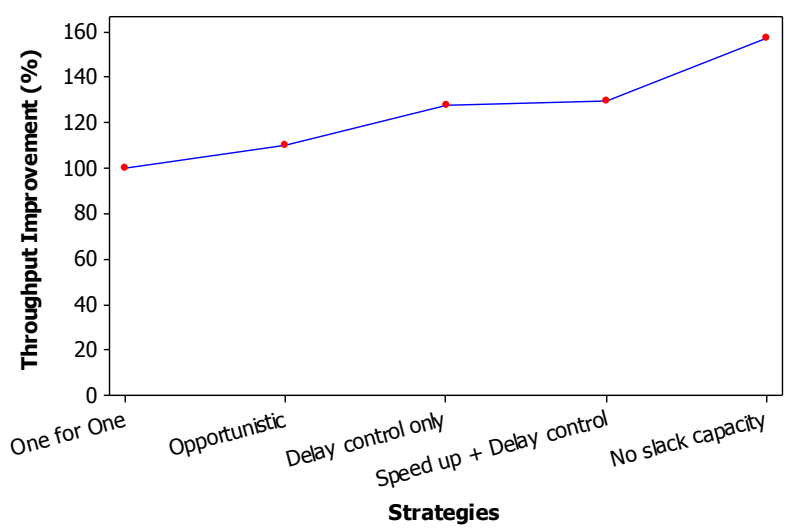

Figure 14. The Impact of Different Strategies on Departures during the Busiest 6-Hour Period. 
Table 3 illustrates the results of the assessment in more detail. We identified several metrics which would help to determine which strategies are more beneficial. The 'Number of Adjustments' column indicates the number of times that an arrival schedule adjustment is made. In the table, the 'Total Induced Delays' column indicates the number of arrival delays that have been induced during the 6-hour period. Values in the 'Average Delay per Arrival' column are seconds in the 'Total Induced Delay' column divided by the number of aircraft that have received a control strategy. The 'Total Advancement in Schedule Time' represents the total seconds that Advance Control is applied to all arrival aircraft. Dividing this value by the number of aircraft that have been controlled with Advance Control is the 'Average Advancement per Arrival" in seconds.' The 'Slack Capacity' is how much wasted capacity in arrival flows there is after adjusting the scheduling by the different control strategies for the entire 6-hour time period.
The results show that the No Slack Capacity Control promises the most benefit out of all three strategies. However, there are some disadvantages to this strategy. For example, once the Scheduled Time of Arrival (STA) is adjusted, the controllers have to condition the arrival aircraft to match the STA. Having to make too many adjustments may lead to an increase in controllers' workload. Also, flexibility is reduced the more tightly controlled the schedules are, which may leave the controllers with little room for error. Hence, both the Delay Control and the Delay and Advance Control strategies may be more promising than the No Slack Capacity approach.

It could be also concluded from the results that using Delay and Advance Control has a greater impact on reducing delay time of the arrivals compared to Delay Control, which could result in a higher reduction of fuel cost.

Table 3. The Results of the Different Strategies' Benefit Assessment (the busiest 6-hour period).

\begin{tabular}{|l|c|c|c|c|c|c|c|}
\hline $\begin{array}{c}\text { Control } \\
\text { Strategies }\end{array}$ & $\begin{array}{c}\text { Number of } \\
\text { Departures }\end{array}$ & $\begin{array}{c}\text { Slack } \\
\text { capacity }\end{array}$ & $\begin{array}{c}\text { Number of } \\
\text { Adjustments } \\
\text { advancement } \\
\text { in Schedule } \\
\text { Time Over } \\
\text { all Aircraft } \\
\text { (seconds) }\end{array}$ & $\begin{array}{c}\text { Advancem } \\
\text { Advancer } \\
\text {-ent per } \\
\text { Arrival } \\
\text { (seconds) }\end{array}$ & $\begin{array}{c}\text { Total } \\
\text { Induced } \\
\text { Delay } \\
\text { (seconds) }\end{array}$ & $\begin{array}{c}\text { Average } \\
\text { Delay per } \\
\text { Arrival } \\
\text { (seconds) }\end{array}$ \\
\hline $\begin{array}{l}\text { One for } \\
\text { One }\end{array}$ & 206 & 6633 & - & - & - & - & - \\
\hline $\begin{array}{l}\text { Opportuni } \\
\text { stic }\end{array}$ & 227 & 3650 & - & - & - & - & - \\
\hline $\begin{array}{l}\text { Delay } \\
\text { control }\end{array}$ & 263 & 2908 & 42 & - & - & 566 & 13.5 \\
\hline $\begin{array}{l}\text { Advance } \\
\text { + Delay } \\
\text { control }\end{array}$ & 267 & 2880 & 47 & -245 & -12.9 & 508 & 18.8 \\
\hline $\begin{array}{l}\text { No Slack } \\
\text { Capacity }\end{array}$ & 324 & 0 & 213 & -1674 & -16 & 1565 & 14.8 \\
\hline
\end{tabular}

To come up with a more accurate estimate of the potential increase in departures and to verify the results from the analysis of the sampled actual traffic data, a Monte Carlo simulation was conducted. We assumed that the arrivals would follow the Poisson process. Hence, the sampled inter-arrival spacing during the selected busiest 6 hours was fitted to the 3parameter Gamma distribution $($ Shape $=5.01$, Scale $=$
10.42, and Threshold = 47.94). One hundred interarrival spacing values were sampled from the fitted distribution and the identified strategies are applied to those samples. The simulation ran 100,000 times. We considered One for One approach as 100\% departure count and construct the plots in Figure 15. As shown in Figure 15, a 10-60\% increase in departures could be expected during the busy hours, which matches 
what we observed in the analysis of the actual traffic data.

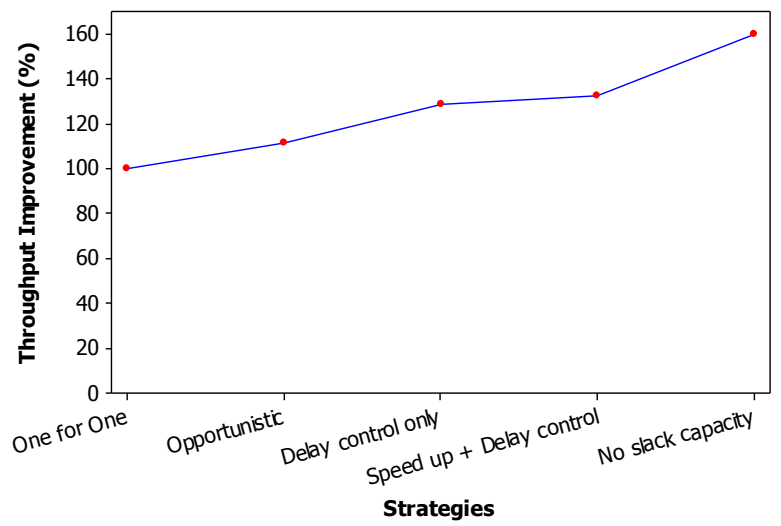

Figure 15. Increase in Departures during Busy Hours - simulated outcome.

\section{Future Work}

In this paper, the benefit assessment was conducted on actual traffic data that was selected to match the most frequently used runway configuration during busy nominal traffic demand in severe clear weather conditions. In future work, other conditions, such as different runway configurations, variable wind conditions, non-homogenous aircraft types, etc., could be examined and tested. The impact of uncertainty in arrival times could be verified as well.

Further cost-benefit analyses could be done to identify a good balance between the additional delay needed between different arrival pairs, the number of additional aircraft the controllers could depart, and the delay in getting the departures off the ground. This analysis could help determine when the proposed concept could be predicted to have the most benefit.

Finally, the feasibility of the concept could be tested in a human-in-the-loop simulation which would prototype the decision support tools, develop operational procedures, and identify any human factors related issues such as workload and needed coordination between controllers. Such a simulation study is being conducted in August, 2014, at the Airspace Operations Laboratory (AOL) at NASA Ames Research Center, and the results will be reported in 2015 .

\section{References}

[1] Welch, Jerry, Steven Bussolari, 2001, Using surface surveillance to help reduce taxi delays, AIAA 2001-4360, Montreal, Quebec, Proceedings of the AIAA Guidance, Navigation \& Control Conference.

[2] Diffenderfer, Paul A., David Osburn, 2012, Improving Throughput at Airports with a Dependency between Arrival and Departure Operations, Herndon, VA, Proceedings of the Integrated Communications Navigation and Surveillance (ICNS) Conference, pp.H1-1.

[3] Diffenderfer, Paul A. Tao Zheng, Payton Gaea, 2013, Automated Integration of Arrival/Departure Schedules, Chicago, Illinois, Proceedings of the Tenth USA/Europe Air Traffic Management Seminar.

[4] Callantine, Todd J., Michael Kupfer, Lynne Martin, Joey Mercer, Thomas Prevot, 2014, SystemLevel Performance Evaluation of ATD-1 GroundBased Technologies, AIAA 2014-2419, Atlanta, GA, Proceedings of the AIAA Aviation Technology, Integration, and Operations Conference.

[5] Department of Transportation, Inspector General Office, 2010, NY flight delays have three main causes, but more work is needed to understand their nationwide effect. Retrieved April 12, 2012 from http://www.oig.dot.gov/library-item/5428

[6] Federal Aviation Administration, Air Traffic Control, April 3, 2014, FAA Order JO 7110.65V, Washington, DC.

[7] Malik, Waqar, Guatam Gupta, Yoon Jung, 2010, Managing Departure Aircraft Release for efficient Airport Surface Operations, AIAA 2010-7696, Toronto, Ontario, Canada, Proceedings of the AIAA Guidance, Navigation, and Control Conference.

\section{Acknowledgements}

The authors would like to thank the lab personnel (particularly, Dr. Bonny K. Parke and Jeffrey Homola for reviewing this paper) at Airspace Operations Laboratory (AOL) at NASA Ames Research center for their great friendship. The authors would also like to thank Dr. Steven J. Landry at Purdue University for his support and insight.

33rd Digital Avionics Systems Conference

October 6-10, 2014 